Historic, Archive Document

Do not assume content reflects current scientific knowledge, policies, or practices. 



\section{PEONIES AND IRIS}

I 927

\section{C. GLENN \\ 2 III Garland Avenue \\ NASHVILLE, TENN.}

TI P A F Y Y

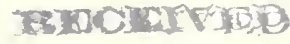
JUL 121929

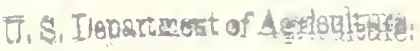

For ten years or more I have collected and grown peonies and iris as a hobby and a diversion from my regular work, which is the teaching of geology in Vanderbilt University.

The collection has grown to such size that the surplus must be disposed of, and hence the following list. The absence of fixed overhead charges has made reasonable prices possible.

Quotations are for three to five eye peony plants and for single rhizomes of iris. The iris may be dug at any time desired, except while blooming. Peonies rvill be dug after September I5th.

Terms are cash with order. Shipment will be by express, collect, unless otherwise specr ified. If shipment is desired by parcel post, please add five cents for each iris or ten cents foeach peony ordered.

No substitutions will be made unless requested. A second choice may be indicated if desired; otherwise your money will be returned. Orders will be filled as long as stock lasts in the order of receipt. Any error will be corrected. No orders for shipment by mail or express accepted for less than $\$ 2.00$.

A collection of 100 mixed iris without names for $\$ 5.00$.

Ten named peonies of my selection, all different, for $\$ 5.00$. 


\section{P E O N I E}

Achille

Acilai Adanson

Adolphe Rousseau

Aksarben....

Alba Sulfurea

Albatre

Albillore

Alexandre Dumas

Alexandriana

Alfred de Musset.-

Alice de Julvecourt

Alma

Alsace-Lorraine

Arietina Baxteri.

Arlequin

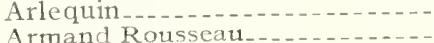

Armandine Mechin _............

Artemise

Asa Gray _........ 100

Auguste Dessert _..... 10.00

Auguste Lemoinier........... .75

Auguste Villaume _........... 1.00

Augustin d'Hour _............ . . .

Aurore

Avant Garde_...... $\quad 3.00$

Aviateur Reymond.......... $\quad 1.50$

Baroness Schroeder _........ $\quad \begin{aligned} & 1.50 \\ & \text { Bayan }\end{aligned}$

Bayadere

Beautc Francaise

Beauty's Mask_..._._._._._. 1.50

Belisaire_............ 1.00

Belle Douaisienne

Beranger .................. 1.00

Berlioz_... $\quad .75$

Bertha_.. 3.00

Blanche Cire... 50

Boadicea. . .

Boule de Neige_...... $\quad .75$

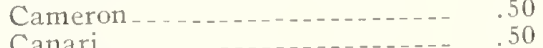

Canari

Candeur

Carnation _. $\quad 50$

Carnea Elegans__._. 50

Charlemagne .50

Charles McKellip _....... 3.00

Charles Sedgwick Minot_..... 4.00

Charles Verdier

Cherry Hill................ 8.00

Chestine Growdy .

Claire Dubois_............. $\quad 1.50$

Clairette_._._._._.

Clara Barton

Claude Gelee
Clemenccaun_...

Comte de Jussier

Comte de Paris

Constant Devred............. 1.00

Coquelin ................... 1.00

Coquette ........ 6.00

Corallina _... 2.00

Decaisne_. $\quad .50$

De Candolle__. $\quad .50$

Decora Elatior

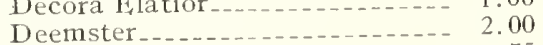

Dclache

Directeur Aubry.
Distinction .............

Dog Rose_.............. 2. 2.00

Dorchester_._. 75

Docteur Boisduval _._..... $\quad .50$

Docteur Boisduran

Dr. H. Barnsby_..... 3.00

Duchess of Portland.......... 2.00

Duchesse de Nemours......... .75

Duchesse d'Orlcans........... $\quad .75$

Duke of Wellington_............ .75

E. C. Shaw 20.00

Edouard Andre

Edulis Superba_.................. 75

E. G. Hill _... 2.00

Elantine

Elie Chevalier - 2.00

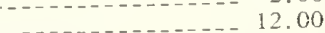

Elizabeth B. Browning _...... 7.50

Elizabeth B. Brine Kelway ....... 1.00

Filwood Pleas

Enchanteresse

Einfant de Nancy

Estafette_._.

Eucharis _.

Eugene Bigot _............ 1.00

Fugene Rcignoux _............ 2.00

Eugene Verdier............ 2.00

Eugenie Verdier............ 1.00

Eureka_.

Evangeline..... 4.00

Evening Glow .............. 4.00

Exquisite _................ 60

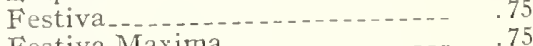

Festiva Maxima _... $\quad .75$

$\begin{array}{lr}\text { Flambeau__._. } & .00\end{array}$

Florence Nightingale_._. _..._. 1.00

Frances Shaylor

Frances Willard_............. 3.00

Francois Ortegat_............ .50

Francois Rousseau_... $\begin{array}{r}3.00 \\ 4.00\end{array}$

Galathee $\ldots \begin{aligned} & 4.00 \\ & 6.00\end{aligned}$

Georgiana Shaylor _._.

Germaine Bigot_.........

Ginette_.
Gisele

Gismonda

Gloire de Charles Gombault _... 1.00

Gloire de Chenonceaux _..... $\quad \begin{array}{r}.75 \\ \text { G }\end{array}$

Gloire de Touraine _._._. $\quad 2.50$

Glory of Summerfield ........ 1.00

Goliath__._.

Grandillora

Grandiflora Carnca Plena_.... $\quad .75$

Grandiflora Rosea ......... $\quad .50$

Graziella__. $\quad .50$

$\begin{array}{ll}\text { Grover Cleveland_... } & 1.00 \\ \text { Harrict Farnsley_......... }\end{array}$

Harrict Farnsley

Henri Laurent . . . . . . . . . . . 50

Hesperus _._. 2.00

$\begin{array}{ll}\text { Humei_._. } & .50 \\ & .50\end{array}$

Humei Carnea _... 500

$\begin{array}{ll}\text { Isoline } & \\ \text { James Kelway } & \end{array}$

Jeanne d'Arc . . . . . . . .50

Jeannott__. _ _ _ _ _ _ 10.00

Jcnny Lind . $\quad \begin{array}{r}50 \\ \text { Jub }\end{array}$

Judge Berry

Kamenokerogoma........
Karl Rosefield _.............. 2.00

Kelway's Glorious _......... 15.00

Kelway's Quee11............... 10.00

Kelways False Queen

La Fayette................ 3.00

La Fee 10.00

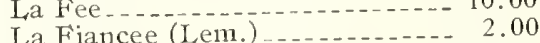

La Fontaine

La Fraicheur. . . . . . . 5.00

Ia France 5.00

La Perle 1.00

La Rosiere................ 1.00

La Sublime . . . . . . . . . . 50

La Tendrcsse_............... 1.00

La Tulipe

Lady Alexandra Duff........ 4.00

Lady Carrington............. 2.00

Lady Derby _._. $\quad .50$

Lady Leonora Bramwell_...... $\quad .00$

Lamartinc (Calot) _........ 1.00

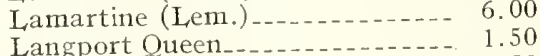

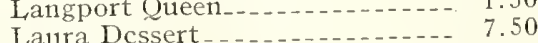

Laura Dcssert_............

L'E 1.00

L'Ecletante

L'Esperance ................. 1.00

Leviathan _................ 50

Libellule _.

Livingstone_................. 1.00

Longfellow _................ _ 5.00

Lora Dexheimer . . . . _ _... 2.50

Lord Kitchener............... 1.00

Louis Van Houtte............ .50

Loveliness _...

Lucida_. 3.00

Lucy E. Hollis .

Madelon_................... 12.00

Marcelle Dessert _............ 2. 2.00

Marchioness of Lansdowne_._._. 2.00

Marechal Vaillant__._._._._. . . 50

Marguerite Gaudichau_..._._._. 5.00

Marguerite Gerard........... 1.00

Marie Crousse _............ 2.00

Maric Dcroux_... $\quad .50$

Marie d'Hour.............. 1.00

Marie Jacquin _.............. 1.00

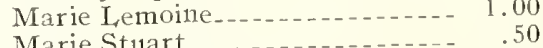

Marie Stuart ............ 2.00

Marquis C. Lagergren_...... 9.00

Mary Brand 3.00

Mary W. Shaylor

Masterpiece

Mathilde de Roseneck.......... 1.00

Maud L. Richardson ......... 2.00

Meissonier

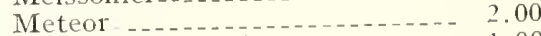

Midnight (Brand) _........... 1.00

Midnight ( $\mathrm{G}$, and $\mathrm{R}$ ) _.. 3.00

Midsumer Night's Dream .... 2.50

Mignon__._. 5.00

Mikado_.................... 2. 2.00

Millais _................. 2.00

Milton Hill _._._. 2.00

Mireillc _____._._. 1.00

Miss Salway _............... 4.00

Mule. Jeanne Gaudichau_...... 5.00

Mlle. Jcanne Riviere . . . . . . . . 3.00

Mlle Julictte Dcssert.......... .50

Mlle. Leonie Calot_._._._._._. 1.00

Mlle. Renee Dessert_._..._._._. .50 
Mlle Rousseau

Mme. Auguste Dessert _..... 2.00

Mme. Auguste Peltereau $\quad .50$

Mme. Barillet Deschamps___._. $\quad .75$

Mme, Benoit Riviere......... 4.00

Mme. Bollet ................... .50

Mme. Boulanger........... 1.50

Mme. Breon _... 50

Mme. Bucquet_........... 1.00

Mme. Chaumy $\quad 50$

Mme. Coste .50

Mme. de Galhau . . . . . . . . . . . .

Mme. de Vatry _... 50

Mme. de Verneville.......... 50

Mme. d'Hour _...... 1.00

Mme. D. Treyeran_...... 2.00

Mme. Ducel

Mme. Emile Dupraz_...... _ 3.00

Mme. Emile Galle_..._._. _. 1.00

Mme. Emile Lemoine........ 1.50

Mme. Forel_............ 75

Mme. Fould _._. 1.00

Mme. Francois Toscanelli ..... 4.00

Mme. Gaudichau _....... 7.50

Mme. Geissler

Mme. Joanne Sallier._._._._. 3.00

Mme. Jules Dessert

Mme. Lebon -

Mme. Lemoine

Mme. Lemoinier. .75

Mme. Loise Mere

Mme. Manehet._...... 400

Mme. Mechin .75

Mme. Muyssart $\quad .50$

Mme. Reignoux _..._._. 1.00

Mme. Savrcau 2.00

Mme. Sehmidt $\quad 1.00$

Mme. Victor Verdier._._._. . . . .

Modele de Perfeetion _........ 1.00

Modeste Guerin

Mons. Barral _............ 1.00

Mons. Bastian Lepage....... .50

Mons. Boueharlataine _........ 1.00

Mons. Dupont_...... .75

Mons. Jules Elie_........ 1.00

Mons. Krelage _..

Mons. Martin Cahuzac ....... 3.00

Mont Blanc_...... 3.00
Mountebank

Mrs. A. C. Ruggles

Mrs. George Bunvard 3.00

Mrs. Key_............. 1.00

Nellie

Ne Plus Ultra 50

Neptune_...

Nigrieans_...

Noemie Demay _..............

Norfolk _...

Nymphe (Des.)

Oetavie Demay

Odette _.

Olfieinalis Alba Mutabilis......

Officinalis Sabina _............ 1.00

O'Fugi _. . . . . . . .

Opal

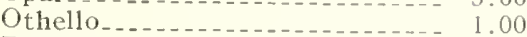

Peregrina Compaeta_......... 2.00

Perfection

Perrette......................... 60

Petite Renee... 2.00

Philippe Rivoire

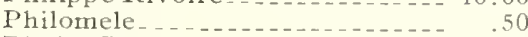

Phobe Cary . . $\quad 5.00$

Phyllis Kelway _.......... 60

Pierre Dessert $\ldots \ldots \ldots$

Picrre Duehartre . . . . . . _.... 1.50

Pierre Reignoux............. 1.50

Poete Frederie Mistral _....... 2.00

Pottsi - .75

Pottsi Alba_................

President Wilson

Primevere 4.00

Priee de Talindyke._._._..... 1.00

Prince of Darkness............. 1.00

Princess Beatrice .......... 50

Princess Maud _._._._._._._. 1.50

Prineess Nicholas Bibeseo...... $\quad .50$

Queen of May_._. 2.00

Queen Victoria_._._. .50

Raehcl (Lem.)

Raoul Dessert . . . $\$$.. $\$ .00$

Raphael ...

Reine Hortense......

Renee Marie_._... 5.00

Richard Carvel $\ldots$

Rita_.. 100
Rosa Bonheur . _ _..._...... 4.00

Rose d'Aniour . . . .

Rosette

R. P. Whitield 2.00

Rubra Superba_..................... 50

Rubra Triumphans

Ruth Brand . _ .

Ruy Blas _................... 1.00

Sapplio - -00

Sarah Bernhardt........... 2.50

Sarah Carstensen............ 5.00

Shabona

Simonne Chevalier............ 1.00

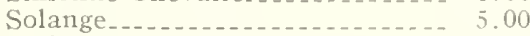

Solfatare -50

Souvenir de Francois Ruitton...- 3.00

Souvenir de Gaspard Calot _... $\quad .75$

Souvenir de l'Expo. Universelle_- 1.00

Souvenir de Louis Bigot....... 5.00

Splendida_................... 3.00

Standard Bearer $\ldots . .00$

Stanley (Crousse) _........... 2.00

Stanley (Kel.)

Summer Day _............... 1.00

Susannc Dessert - 1.50

Suzette........................ 2.

Tamatbako_................ 15.00

Teniere- 50

The Moor................ 2.00

Thercse_...................... 4.00

Tourangelle_...

Triomphe de l'Exp. de Lille_ _ _ .50

Triomphe du Nord _......... .50

Umbellata Rosea ............. . . 75

Venus.

Vesuve

Victoire de la Marne....... 3.00

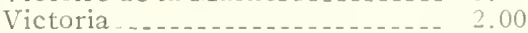

Ville de Nancy ................. 50

Valter Fayon 3.00

Welcome Guest. ........... 2.00

$\begin{array}{ll}\text { White Lady } & 6.00\end{array}$

William F. Turner___._____ 4.00

William Penn _._._._._._. I.00

Yeso 4.00

Zoe Calot

\section{R I S}

\begin{tabular}{|c|c|c|c|c|c|}
\hline BEARDED IRIS & & Aurea $\ldots \ldots \ldots$ & .25 & 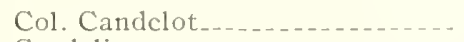 & .50 \\
\hline Afterglow $\ldots \ldots \ldots$ & .75 & $\begin{array}{l}\text { Aurora } \ldots \ldots \\
\text { Azure } \ldots \ldots\end{array}$ & .50 & $\begin{array}{l}\text { Cordelia } \\
\text { Cordon Bleu }\end{array}$ & 50 \\
\hline Albert Victor & .25 & Ballerine & 1.00 & Corrida_... & .50 \\
\hline Aleazar...... & .50 & Black Prince . . . . & 1.00 & Crepuseulc $\ldots \ldots \ldots$ & .50 \\
\hline Amas $\ldots \ldots$ & .25 & Blue Bird $\ldots \ldots$ & .75 & Cretonnc $\ldots \ldots \ldots$ & .50 \\
\hline Ambassadeur . . & 1.00 & Bosniamac . . . . . & .50 & 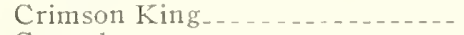 & .25 \\
\hline Ambigu_... & .50 & B. Y. Morrison $\ldots$ & 1.00 & Crusader $\ldots \ldots \ldots$ & .50 \\
\hline Amethyst & .50 & Camelot $\ldots \ldots$ & .50 & Dalila $\ldots \ldots \ldots$ & .50 \\
\hline Angelo & 2.00 & Caprice $\ldots \ldots \ldots$ & .25 & Dalmarius . & .25 \\
\hline Anne Leslie & 1.00 & Carthusian $\ldots \ldots \ldots$ & .50 & 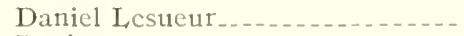 & 1.00 \\
\hline Archeveque & .50 & Caterina & .50 & Darius_................. & \\
\hline Argynnis $\ldots \ldots \ldots \ldots \ldots$ & 2.00 & Cccil Minturn & .75 & Dawn $\ldots$ & \\
\hline Arlequin $\ldots \ldots \ldots$ & .50 & Celestc $\ldots \ldots$ & .25 & Dejazet & \\
\hline Arnols. & .25 & Chatelet & 2.00 & Delicatissima & 1.00 \\
\hline Asia_n & 3.00 & Clara Curtis $\ldots \ldots \ldots$ & .50 & Diadcm & .50 \\
\hline A thene $\ldots \ldots$ & .50 & Cluny $\ldots \ldots \ldots \ldots$ & .50 & Dianc $\ldots \ldots \ldots \ldots$ & .50 \\
\hline
\end{tabular}


Donna Nook

Dora Longdon

Dorine

Dorothea ...................

Dr. Bernice ................

Dream

Dusky Maid _... _. _ _. _ _

Edouard Michel ..............

E. H. Jenkins

Emmeline . . . .

Empress of India

Etenard

Fairy -

Firmament ................

Flavescens

Florence Wells_................

Florentina Alba .................

Gaite_-_._._.

Gajus_...

Gold Crest $\ldots \ldots \ldots$

Gov. Hughes_...

Gracchus_...

Grand Mogul

Gules_-

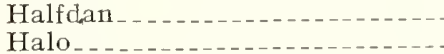

Hautefeuille

Hector $\ldots \ldots$

Helge Majesty

Hugo

Igouf

Innocenza .

Iris King

Ivanhoe

Jean Sisley

Junon

Kathryn Fryer

Kharput_...................

Knysna

Kochi La Neige $\ldots_{\text {L }}$

Lady Byng....................

Lady Foster .................

Lady Lilford

Lady Seymour

L'Avenir

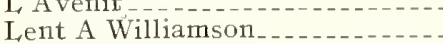

Leone Trenance.................

Leonidas
Lepinoux.-.

Lever rier.

Lionel Millet. ..............

Lohengrin......................

Lord of June ..................

Loreley_.....................

Loute

Lurida Mady Carriere

Magnifica
1.00
Magot

Maiden's Blush

MaMie . . . . . . .

Mandraliscae _..............

Margaret Moor _.

Marigny

Mary Garden . . . . . . . . . . .

Mary Williamson.

M. Cornault

Medrano

Megas.-

Mercedes

Merlin

Mildred Presby

Miralba

Miranda...........................

Miriam.

Miss Eardley

Miss Willmott

Mlle. Schwartz ...............

Mme. Chereau

Mme. Pacquitte

Mme. Truffaut_.............

Moliere_..............

Moncey _. _. . . . . .

Monhassan_..._.............

Monsignor.

Morwell

Mrs. Allan Gray _...........

Mrs. H. Darwin

Mrs. Neubronner..............

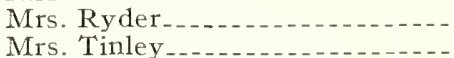

Mrs. Walter Brewster..........

Nancy Orne._._............

Nazarin

Neptune

Nine Wells.............................

O. Perthuis . . . . . .

Opera

Oriflamme...

Pallida Dalmatica...........

Pallida Speciosa . . . . . . . . . .

Parc de Neuilly

Parisiana

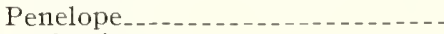

Perfection

Phyllis Bliss..................

Prestige

Princess Victoria Louise........

Prosper Laugier..............

Prospero .....................

Quaker Lady

Queen Alexandra...

Queen of May
Raffet.

Regan_...

Reticulata Alba

Rhein Nixe

Ricardi Fonce_.............. 1.00

Richard II

Ring Dove.... 50
Rodney........................ 50

Rose Unique .................. . . . 25

Roseway

Sambucina_._._. . 25

Shakespeare_._._._._._._._. .25

Shalimar.

Shekinah _ _ 1.00

Shelford Chieftain

Sherbert__._...........

Simon Vaissiere

Sindjkha_-__._. .50

Sirdar...........

Soliman _ $\quad .50$

Sumercoates $\quad .50$

Souv. de Delagrange _........ .50

$\begin{array}{lr}\text { Souv, de Mme Gaudichau _. . . . } & 1.00 \\ \text { Speciosa }\end{array}$

$\begin{array}{ll}\text { Speciosa_-25 } & .25 \\ \text { Spectabilis } & .50\end{array}$

Stamboul_...

Sudan

Suzanne Autissier. ........... 1.00

Sweet Lavender_........... 1.00

Tamar_... 50

Tamerlan_... . 50

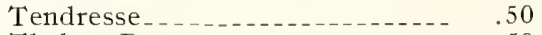

Thelma Perry .50

Thorbecke

Tom Tit

Toreador_... 50

Tristram ........................

Trojana_._. 50

Troost_...

Turco _._._. 50

Valery Mayet_... 1.00

Veloute _._. $\quad .50$

Victorine

Vieil Or. 50

Violacea Grandiflora_.......... . . . 25

Violet Episcopal

Virginia Moore........... 1.00

Walhalla___ .25

William Marshall

W. J. Fryer $\ldots 1.00$

Wyomissing .25

Yvonne Pelletier...

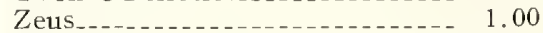

Zouave

Zwanenburg

.50

\section{BEARDLESS IRIS}

Fulva

Fulvala_- 1.00

Mandshurica $\quad .50$

Monnieri...... 50

Ochroleuca_. 50

Pseudacorus _................ . . .50

Sib. Mrs. Perry

Sib. Mrs. Saunders

Sib. Peggy Perry ............. . .50

Sib. Perry's Blue

Sib. Perry's Emperor

Sib. Snow Queen .25

Sib. Superba_.... 25

Spuria Alba

Tectorum _.... 